\section{Fine structural detection of calcium ions by photoconversion}

\author{
V. Poletto, ${ }^{1}$ V. Galimberti, ${ }^{2}$ G. Guerra, ${ }^{3}$ \\ V. Rosti, ${ }^{1}$ F. Moccia, ${ }^{4}$ M. Biggiogera ${ }^{2}$ \\ ${ }^{1}$ Centro per lo Studio e la Cura della \\ Mielofibrosi, Laboratori Sperimentali di \\ Ricerca, Area Biotecnologie, Fondazione \\ IRCCS Policlinico San Matteo, Pavia \\ ${ }^{2}$ Laboratorio di Biologia Cellulare e \\ Neurobiologia, Dipartimento di Biologia e \\ Biotecnologie, Università di Pavia \\ ${ }^{3}$ Dipartimento di Medicina e Scienze della \\ Salute, Università del Molise, \\ Campobasso \\ ${ }^{4}$ Laboratorio di Fisiologia Generale, \\ Dipartimento di Biologia e Biotecnologie, \\ Università di Pavia, Italy
}

\section{Abstract}

We propose a tool for a rapid high-resolution detection of calcium ions which can be used in parallel with other techniques. We have applied a new approach by photo-oxidation of diaminobenzidine in presence of the emission of an excited fluorochrome specific for calcium detection. This method combines the selectivity of available fluorophores to the high spatial resolution offered by transmission electron microscopy to detect fluorescing molecules even when present in low amounts in membranebounded organelles. We show in this paper that Mag-Fura 2 photoconversion via diaminobenzidine oxidation is an efficient way for localizing $\mathrm{Ca}^{2+}$ ions at electron microscopy level, is easily carried out and reproducible, and can be obtained on a good amount of cells, since the exposure in our conditions is not limited to the direct irradiation of the sample via an objective but obtained with a germicide lamp. The end product is sufficiently electron dense to be detected clearly when present in sufficient amount within a membrane boundary.

\section{Introduction}

The ultrastructural localization of a molecule requires that a certain degree of electrondensity, resulting in adequate electron scattering, be present in sufficient amount. Binding of heavy atoms can be accomplished easily with regards to proteins, nucleic acids and other cellular components, in some cases with a high degree of specificity. ${ }^{1}$ However, the subcellular localization of ions is a challenge, since in many cases ions are diffusible and not easily kept in place after the procedures of sample preparation for electron microscopy (EM). Many ions can offer enough scattering in spite of not being heavy metals, such as manganese, while others necessitate of blocking or precipitating techniques to be visualized. In particular, calcium ions are easily diffusible inside the cell, and even when proteinbound are not easily detectable. The pioneering paper by Tandler et al. ${ }^{2}$ demonstrated the presence of calcium ions by EM via pyroantimonate precipitation, with a good yield of the final product. The end product of the reaction, however, is probably overrated, since the technique is capable of stabilizing and precipitating calcium and other bivalent cations, such as magnesium, as proved by electron spectroscopic imaging (ESI) analysis. ${ }^{3}$

Since their introduction by Tsien and coworkers, ${ }^{4}$ calcium studies have used specific $\mathrm{Ca}^{2+}$-sensitive fluorochromes, such as Fura-2, Indo-1 and Fluo-4, coupled to wide-field digitized video fluorescence microfluorimetry and confocal microscopy to image acute elevations in intracellular $\mathrm{Ca}^{2+}$ concentration $\left(\left[\mathrm{Ca}^{2+}\right]_{\mathrm{i}}\right)$ with high spatio-temporal resolution. ${ }^{5,6}$ Cytosolic and nuclear $\mathrm{Ca}^{2+}$ signals regulate a multitude of vital cellular processes, such as gene transcription, cell cycle progression, cytoskeletal and chromatin remodelling, and migration. ${ }^{7-9}$ However, proper $\mathrm{Ca}^{2+}$ handling within the endoplasmic reticulum (ER), the most abundant intracellular $\mathrm{Ca}^{2+}$ reservoir, ${ }^{10}$ is also crucial for cell-fate decisions. The steadystate ER $\mathrm{Ca}^{2+}$ level $\left(\left[\mathrm{Ca}^{2+}\right]_{\mathrm{ER}}\right)$ does not only control synthesis, folding, export and trafficking of proteins, ${ }^{10,11}$ but it also fuels mitochondrial bioenergetics thereby suppressing autophagy. ${ }^{12}$

Low affinity $\mathrm{Ca}^{2+}$ indicators, such as MagFura 2, and genetically-encoded probes, such as the bioluminescent protein aequorin and the GFP-based fluorophores camgaroos, pericams, and cameleons, have been devised to assess ER $\mathrm{Ca}^{2+}$ levels. ${ }^{13,14}$. Nevertheless, these sophisticated tools do not provide any reliable information on $\mathrm{Ca}^{2+}$ distribution within the heterogeneous structure of the ER. We propose in this paper a tool for a rapid high-resolution detection of calcium ions which can be used in parallel with other techniques. We have applied a new approach to the detection of intracellular calcium ions. Maranto ${ }^{15}$ and Sandell and Masland ${ }^{16}$ have proposed the possibility of photoconverting fluorochromes into an electrondense end product. The technique utilizes the photo-oxidation of diaminobenzidine (DAB) in presence of the emission of an excited fluorochrome, and it has been shown to be efficient on different dyes and conditions. ${ }^{17,18}$ This method combines the specificity of available fluorophores to the high spatial resolution offered by transmission EM to detect fluorescing molecules
Correspondence: Marco Biggiogera, Laboratorio di Biologia Cellulare e Neurobiologia, Dipartimento di Biologia e Biotecnologie, Università di Pavia, Via Ferrata 9, 27100 Pavia, Italy.

Tel. +39.0382.986322 - Fax: +39.0382 .986325 .

E-mail: marco.biggiogera@unipv.it

Key words: Ultrastructural cytochemistry; calcium ions; photoconversion; Mag-Fura 2.

Received for publication: 17 June 2016. Accepted for publication: 5 July 2016.

This work is licensed under a Creative Commons Attribution-NonCommercial 4.0 International License (CC BY-NC 4.0).

(C) Copyright V. Poletto et al., 2016

Licensee PAGEPress, Italy

European Journal of Histochemistry 2016; 60:2695 doi:10.4081/ejh.2016.2695

even when present in low amounts in membrane-bounded organelles. The present manuscript sought to exploit DAB photo-oxidation of Mag-Fura 2 for visualizing $\mathrm{ER} \mathrm{Ca}^{2+}$ distribution in HeLa cells, which are among the most widespread model cell lines used for studying intracellular $\mathrm{Ca}^{2+}$ signaling.

\section{Materials and Methods}

\section{Cell culture}

Hela cells have been cultured in Iscove's Modified Dulbecco's Medium (IMDM, Lonza, Basel, Switzerland) supplemented with $10 \%$ fetal bovine serum, $2 \%$ L-Glutamine, $1 \%$ penicillin and streptomycin, at $37^{\circ} \mathrm{C}$ air atmosphere with $5 \% \mathrm{CO}_{2}$. After reaching $90 \%$ confluence, cells have been plated in 6 wells cell culture plate ( $1 \times 10^{5}$ cells per well), or in chamber slides for staining and microscopic examination ( $8 \times 10^{3}$ cells per well). The day after seeding, when cells reached about $90 \%$ confluence, the medium has been removed from the wells and replaced with three solutions, one for each experimental condition. The solutions, different in $\mathrm{Ca}^{2+}$ concentration, are the following: physiological salt solution (PSS), with the following composition: $150 \mathrm{mM} \mathrm{NaCl}, 6 \mathrm{mM} \mathrm{KCl}$, $1.5 \mathrm{mM} \mathrm{CaCl}_{2}, 1 \mathrm{mM} \mathrm{MgCl}_{2}, 10 \mathrm{mM}$ Glucose, and $10 \mathrm{mM}$ Hepes; $\mathrm{Ca}^{2+}$-free solution $\left(0 \mathrm{Ca}^{2+}\right)$, where $\mathrm{Ca}^{2+}$ has been substituted by $2 \mathrm{mM}$ $\mathrm{NaCl}$, and $0.5 \mathrm{mM}$ EGTA was added, whereas the solution with $\mathrm{Ca}^{2+}$ more concentrated is similar to PSS in composition except for 120 $\mathrm{mM} \mathrm{NaCl}$ and $20 \mathrm{mM} \mathrm{CaCl}$. All the solutions have been brought to $\mathrm{pH} 7.4$ with an osmolality of $338 \mathrm{mmol} / \mathrm{kg}$, measured with an osmometer (Wescor 5500; Wescor, Inc., Logan, UT, USA). 


\section{Intracellular $\mathrm{Ca}^{+}$labeling and diaminobenzidine-photoconversion}

Intracellular $\mathrm{Ca}^{2+}$ deposits have been marked by fluorescent dye-based calcium indicator Mag-Fura-2, acetoxymethyl (AM) ester UV light-excitable (Life Tchnologies, Carslbad, CA, USA), added to the three calcium solutions to obtain a final concentration of $5 \mu \mathrm{M}$. Cells have thus been incubated at $37^{\circ} \mathrm{C}$ in the dark, for $1 \mathrm{~h}$. Cells cultured on chamber slides, and treated as hereto described, have been analyzed at fluorescence microscopy. An Olympus BX51 microscope (Olympus Italia Srl, Segrate, Italy) was used under the following conditions: 100-W Hg lamp as the excitation source, 330to $385-\mathrm{nm}$ excitation filter (excf), 400-nm dichroic mirror (dm), and 420-nm barrier filter (bf); 100× Olympus UplanFl oil-immersion objective lens (NA 1.25). Images were recorded with an Olympus Magnifier digital camera system, and stored by the Olympus CellF software.

Instead, cells in plates have been fixed at room temperature for 20 minutes with a fixative solution (2\% paraformaldehyde and $1 \%$ glutaraldehyde in PBS). Meanwhile, a solution with $\mathrm{DAB}$ has been freshly prepared as follows: $20 \mathrm{mg}$ of DAB (Sigma Aldrich, St. Louis, MO, USA) were dissolved in $1 \mathrm{ml}$ DMSO, briefly vortexed and then brought to $30 \mathrm{~mL}$ with PBS. Thereafter, cells have been washed twice with PBS and incubated for $3 \mathrm{~h}$ under a germicidal UV lamp (Sankyo Denki, Kanagawa, Japan; G30T8, UV-C) with $2 \mathrm{~mL}$ DAB solution which is replaced with fresh solution every half an hour.

In parallel, some cells were incubated in the absence of Mag-Fura 2, treated with DAB and processed as above. Moreover, to exclude a possible interference by aldehyde groups, some slides were fixed and then treated with $0.5 \mathrm{M} \mathrm{NH} \mathrm{Nl}_{4} \mathrm{Cl}$ PBS for $30 \mathrm{~min}$ at $4^{\circ} \mathrm{C}$, then incubated with $\mathrm{DAB}$ and processed as above.

\section{Transmission electron microscopy}

Fixed and photoconverted cells have been washed twice with distilled water and postfixed with $2 \%$ osmium tetroxide in water for $1 \mathrm{~h}$, at room temperature. Once the osmium was removed and the cells rinsed several times with distilled water, cells have been scraped, centrifuged discarding supernatant, dehydrated in a graded series of ethanol and embedded in LRWhite resin (Sigma Aldrich). Ninety nm ultrathin sections have been cut by a Reichert ultramicrotome, transferred to grids without membrane and visualized by a Zeiss EM900 transmission electron microscope operating at $80 \mathrm{kV}$.

\section{Results}

Calcium labeling with Mag-Fura 2 results in highly fluorescent staining mainly localized in the cytoplasm. After incubation of the cells with $20 \mathrm{mM} \mathrm{Ca}^{2+}$ for $1 \mathrm{~h}$, the signal is present in large vesicles in the cytoplasm and around the cell nucleus, as well as in elongated structures probably related to mitochondria (Figure 1a). The faint signal visible in the nucleus is very probably due to the superposition of the cytoplasmic layer surrounding it. When the cells are incubated in PSS, the signal is lower (Figure 1b) and mostly present in the perinuclear vesicles, while the cytoplasmic signal far from the nucleus somehow disappears. In the last condition, without calcium and in the presence of EGTA, some labeling is still visible, although to a much reduced level (Figure 1c). This continuous presence of $\mathrm{Ca}^{2+}$ represents very probably the last ions the cell cannot allow to lose, a sort of surviving threshold concentrations. Supplementary Figure 1 confirms that altering extracellular $\mathrm{Ca}^{2+}$ concentrations results in a consequent change in $\mathrm{ER} \mathrm{Ca}^{2+}$ homeostasis, which may have important consequences also on cytosolic $\mathrm{Ca}^{2+}$ dynamics. At EM level, the controls did not show any electron dense precipitates, both in the absence of the fluorochrome or after aldehyde blockade (not shown); consequently, the end product obtained in the Mag-Fura 2 stained cells is due to the fluorochrome photoconversion. The DAB photoconversion is an efficient way to stabilize the ions and to visualize them. It must be underlined here that we observe a thin section, 60-80 nm thick, corresponding, consequently, only to a fraction of the fluorescent signal.

After PSS incubation, the cells show a distinctive positivity (Figure 2a). In some cases it is possible to see the presence of precipitates without the direct association with smooth or rough ER (SER or RER, respectively) membranes, likely related to unbound $\mathrm{Ca}^{2+}$ ions (Figure 2b), and electron dense material inside the vesicles is also positive (Figure 2c). A possible suggestion of $\mathrm{Ca}^{2+}$ internalization or extrusion is shown in Figure 2 d,e, where the deposits are present on the outer membrane. When the cells are incubated without calcium and in the presence of EGTA, the deposits are extremely reduced, although still present on the outer cell membrane, suggesting the presence of an extruding mechanism (Figure 2f). On the contrary, the diffuse, low density end product is almost absent from the cytoplasm.

At higher $\mathrm{Ca}^{2+}$ concentration in the medium (Figure 3a), the dark precipitate is detectable on the inner membrane of cytoplasmic vesicles possibly pertaining to SER, and in discrete foci with no apparent association with vesicles or membranes. The deposits are present within electron dense vesicles (Figure 3b) and electron lucent structure, in some cases associated, and suggesting the idea of pas- sage of ions from one compartment to another. In other cases, DAB-positive material is present on the membranes of complex systems such as multivesicular bodies (Figure $3 c$ ) or on the outer membranes of cytosolic vesicles (Figure 3d). Interestingly, calcium deposits are detectable on the outer cell membrane (Figure 3e) in spots, localized and separated, suggesting the presence of calcium
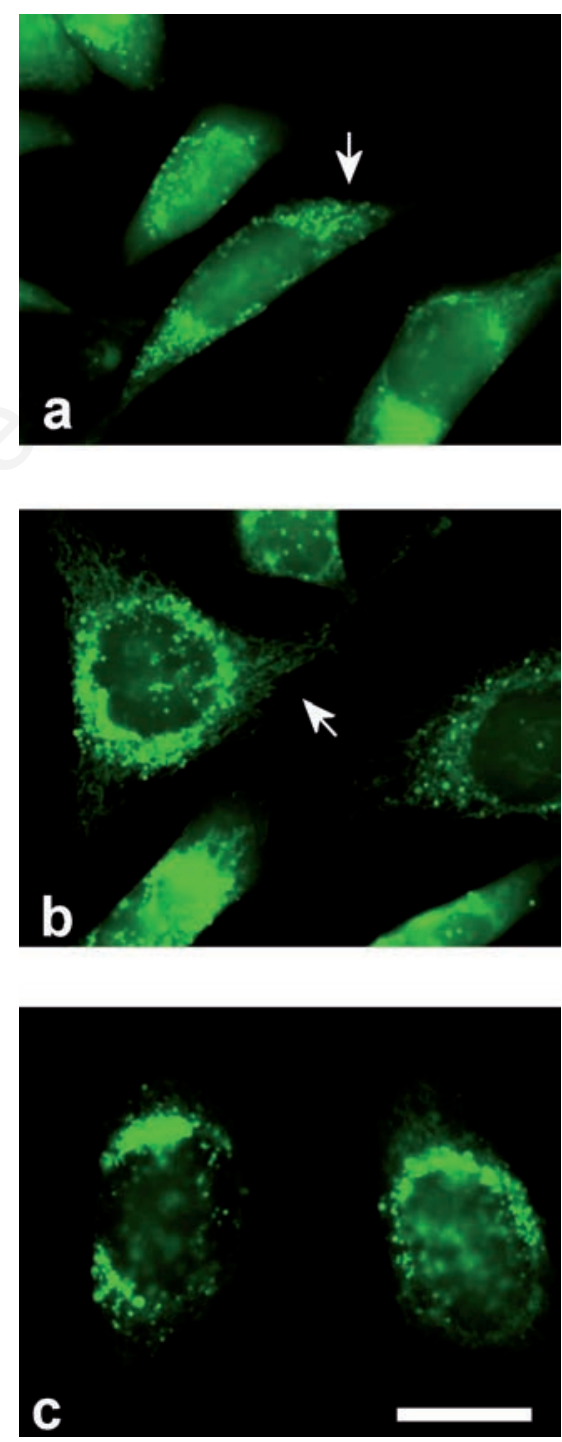

Figure 1. Detection of $\mathrm{Ca}^{2+}$ in fluorescence after Mag-Fura 2 staining. a) $\mathrm{High}^{\mathrm{Ca}^{2+} \text { con- }}$ centration; the labeling is present in the cytoplasm within vesicles as well as in filamentous structures (arrow) probably related to ER. b) Normal $\mathrm{Ca}^{2+}$ concentration; the vesicles are more evident near the nuclear envelope, while the cytoplasm is less labeled with the exception of the filamentous structures (arrow). c) When $\mathrm{Ca}^{2+}$ is omitted from the medium, only the perinuclear vesicles are labeled. Scale bars: $10 \mu \mathrm{m}$. 
channels. In Figure 3f, another example of surface labelling of calcium ions is presented. The end product of the DAB photoconversion is a speckled, dense precipitate of varying size. However, another abundant end product is present as small sized dots, showing a markedly lower electrondensity (Figure 3f, inset) and a widespread distribution.

\section{Discussion}

We show in this paper that Mag-Fura 2 photoconversion via DAB oxidation is an efficient way for localizing $\mathrm{Ca}^{2+}$ ions at EM level. Moreover, this could be considered also as a means of stabilizing their presence, thus limiting the loss of ions. As for the method, photoconversion is easily carried out and reproducible, can be obtained on a good amount of cells, since the exposition in our conditions is not limited to the direct irradiation of the sample via an objective but obtained with a germicide lamp.

The end product is sufficiently electron dense to be detected clearly when present in sufficient amount within a membrane boundary. In these conditions, the blackness of the product stands out from the background. In our conditions, in fact, $\mathrm{Ca}^{2+}$ ions are immediately detectable within the vesicles and the tubules of the SER, while it is much more difficult to see their presence when in the cytosol, due to their low electrondensity. This is a clear advantage over the conventional imaging of MagFura 2 fluorescence that must be removed by the cytosol in order to study truly ER signals. ${ }^{19,20}$ This is achieved by permeabilizing the plasma membrane with brief applications of antibiotics or detergents, but this procedure may destroy intracellular signaling pathways, dilute cytosolic modulators of $\mathrm{ER} \mathrm{Ca}^{2+}$ release/sequestration, and disassemble the cytoskeletal elements that maintain the con-
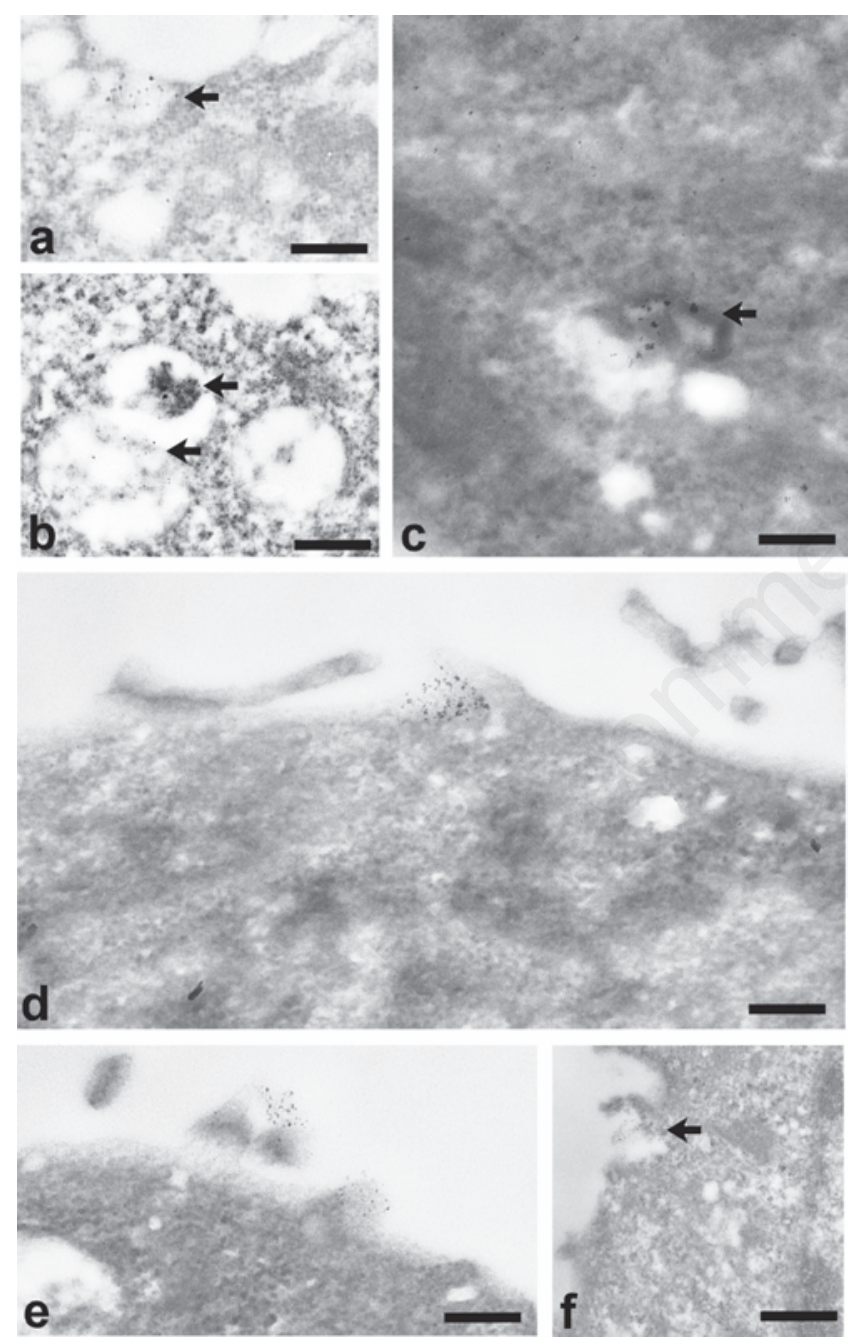

Figure 2. Endoplasmic reticulum photoconversion. When the cell culture medium contains normal $\mathrm{Ca}^{2+}$ concentrations, the dense precipitates can be visualized in the cytoplasm (a) in the vicinity of vesicles, or within them (b) or as being released in open structures (c). At the cell surface (d,e) the deposits suggest a process of extrusion/incorporation of $\mathrm{Ca}^{2+}$ ions. In (f) very few precipitates are present when $\mathrm{Ca}^{2+}$ is not present in the culture medium. Scale bars: a-c) $200 \mathrm{~nm}$; d-e) $100 \mathrm{~nm}$; f) $200 \mathrm{~nm}$.

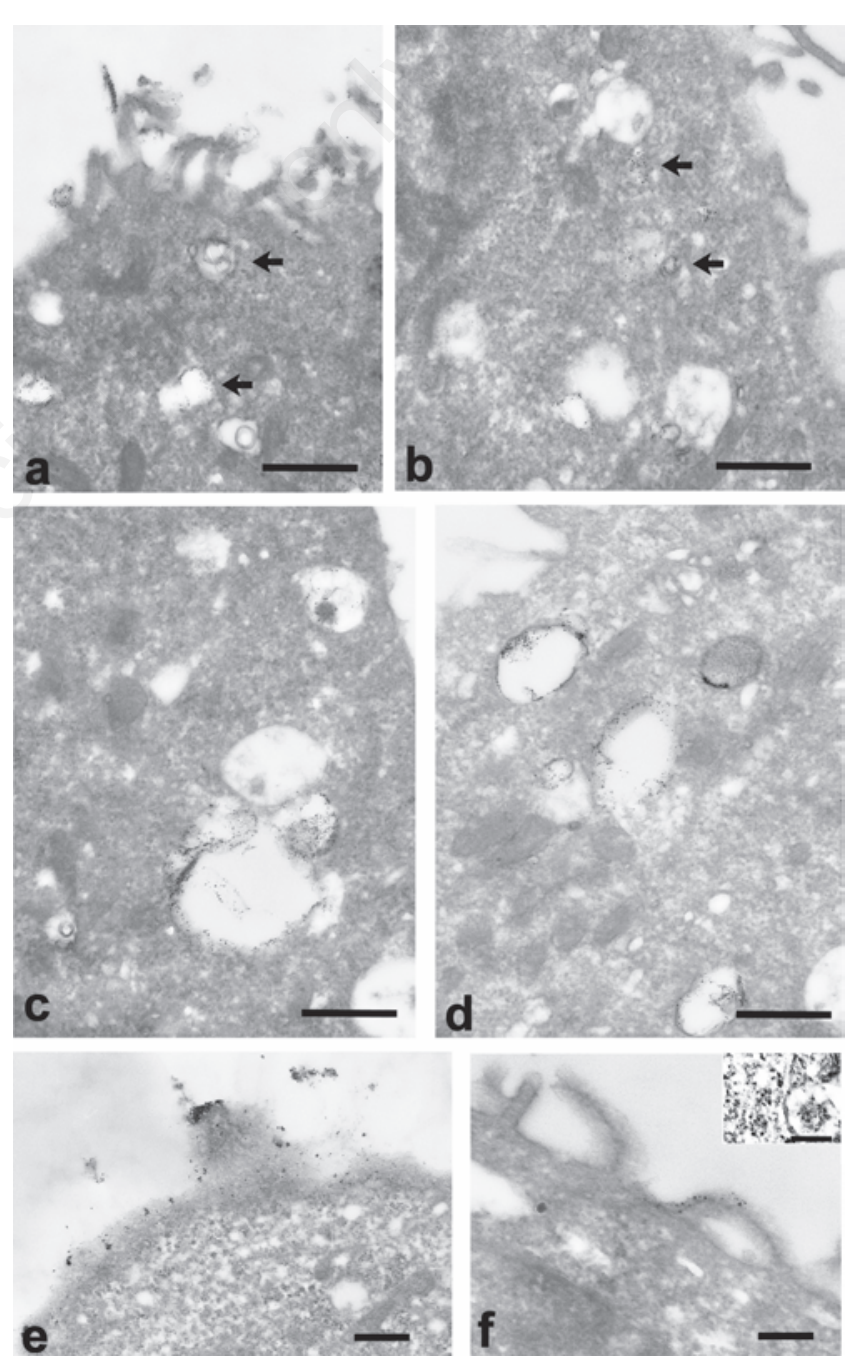

Figure 3. Endoplasmic reticulum photoconversion. At high $\mathbf{C a}^{2+}$ concentration, the end product can be found both in vesicular structures (arrows) as well as on the outer membrane structures (a) or in the cytoplasm nearby vesicles (b, arrows). The end precipitates are also present lining the inner membrane of the ER-derived structures (c, d). Interestingly, the staining is present on the membrane in discrete spots at regular distance (e) or on membrane protrusions (f) as well as diffuse precipitates in the cytoplasm (inset). Scale bars: a-d) $500 \mathrm{~nm}$; e-f) $100 \mathrm{~nm}$; finset, $100 \mathrm{~nm}$. 
tact between cell membrane and ER. Alternatively, the whole-cell configuration of the patch-clamp technique could be exploited to remove the dye, but this experimental approach is rather expensive and challenging, requires highly skilled electrophysiologists and has a quite low throughput. The final yield of photoconverted Mag-Fura 2 is in direct relation with the amount of Ca present in the sample considered. In our condition, the Ca-enriched medium gave a higher end product staining than the normal physiological conditions or than $\mathrm{Ca}$ deprivation.

It is interesting that in several cases, dense precipitates could be found in areas suggesting the local accumulation of $\mathrm{Ca}^{2+}$ nearby the mouth of plasmalemmal $\mathrm{Ca}^{2+}$ channels. Accordingly, such precipitates are evident when extracellular $\mathrm{Ca}^{2+}$ concentration is increased, which is consistent with the notion that constitutive $\mathrm{Ca}^{2+}$ inflow from the extracellular milieu is enhanced. This unexpected outcome of DAB photoxidation is extremely relevant in light of the master role served by local $\mathrm{Ca}^{2+}$ microdomains generated by extracellular $\mathrm{Ca}^{2+}$ influx, which regulate cell functions as diverse as cycle progression, proliferation, migration, nitric oxide production, and adenylyl cyclase activity. ${ }^{7,21}$ These findings will be further explored in a work now in progress.

One thing that should be underlined here is that we could not visualize the SER tubule filled with the end product. This could be due to three different causes: i) $\mathrm{Ca}^{2+}$ can be dispersed (lost) in the conditions used here; ii) local intraluminal $\mathrm{Ca}^{2+}$ concentration is less than expected (which is less probable as it is unlikely to be lower than $100 \mu \mathrm{M}$ ); or iii) DAB photoconversion is not efficient enough for Mag-Fura.

By comparing fluorescence images with EM micrographs, it is clear the difference in the signal coming from vesicles and the elongated filamentous structures, the latter being much less fluorescent.

The relative low yield when comparing fluorescence images is definitely related to the extremely lower amount of end product which can be found within a $60-80 \mathrm{~nm}$ thin section in relation to the fluorescence originating from the whole cell. In addition, albeit $\left[\mathrm{Ca}^{2+}\right]_{\mathrm{ER}}$ fluctuates between 100 and 500 $\mu \mathrm{M},{ }^{20} \mathrm{Ca}^{2+}$ accumulates in specific regions of the $\mathrm{ER}^{22}$ that may be missed in our sections. Moreover, releasable $\mathrm{Ca}^{2+}$ is actually trapped by oligomers of the $\mathrm{Ca}^{2+}$-binding proteins calnexin and calreticulin: these form a concealed $\mathrm{Ca}^{2+}$ source that is not in rapid equilibrium with the $\left[\mathrm{Ca}^{2+}\right]_{\mathrm{ER}}$, but is easily accessible to $\mathrm{Ca}^{2+}$-releasing channels upon extracellular stimulation. ${ }^{23}$ Thus, the lack of SER tubules or cisternae filled with the end prod- uct of the photo-conversion, i.e., $\mathrm{Ca}^{2+}$, is not surprising. Finally, the photoconversion process seems to be efficient, and gives an end product more reasonable than the precipitation technique utilized, for instance, by Tandler et al. ${ }^{2}$ In the latter case, an important amount of precipitates were found in the cell and especially in the nucleus; our method gives a much finer end product, and this allows a superior resolution and localization.

In conclusion, this method is a reliable and efficient technique for stabilizing and visualizing $\mathrm{Ca}^{2+}$ ions at EM. It gives a high resolution end product thus allowing the fine structural $\mathrm{Ca}$ localization. In principle, this approach could provide important qualitative information about the amount and sub-cellular distribution of calcium ions both in cultured cells and, more importantly, in ex vivo samples, which are far less amenable to high resolution $\mathrm{Ca}^{2+}$ imaging of $\mathrm{ER} \mathrm{Ca}^{2+}$ levels.

\section{References}

1. Hayat H. Stains and cytochemical methods. New York, London, Plenum Press, 1993.

2. Tandler CJ, Libanati CM, Sanchis CA. The intracellular localization of inorganic cations with potassium pyroantimonate. Electron microscope and microprobe analysis. J Cell Biol 1970;45:35566 .

3. Boutinard Rouelle-Rossier V, Biggiogera M, Fakan S. Ultrastructural detection of calcium and magnesium in the chromatoid body of mouse spermatids by electron spectroscopic imaging and electron energy loss spectroscopy. J Histochem Cytochem 1993;41:1155-62.

4. Tsien RY, Pozzan T, Rink TJ. T-cell mitogens cause early changes in cytoplasmic free $\mathrm{Ca} 2+$ and membrane potential in lymphocytes. Nature 1982;295:68-71.

5. Bootman MD, Rietdorf K, Collins T, Walker S, Sanderson M. Ca2+-sensitive fluorescent dyes and intracellular $\mathrm{Ca} 2+$ imaging. Cold Spring Harb Protoc 2013;2013:83-99.

6. Dragoni S, Laforenza U, Bonetti E, Lodola F, Bottino C, Berra-Romani R, et al. Vascular endothelial growth factor stimulates endothelial colony forming cells proliferation and tubulogenesis by inducing oscillations in intracellular $\mathrm{Ca} 2+$ concentration. Stem Cells 2011;29:1898907.

7. Berridge MJ, Bootman MD, Roderick HL. Calcium signalling: dynamics, homeostasis and remodelling. Nat Rev Mol Cell Biol 2003;4:517-29.
8. Clapham DE. Calcium signaling. Cell 2007;131:1047-58.

9. Moccia F, Tanzi F, Munaron L. Endothelial remodelling and intracellular calcium machinery. Curr Mol Med. 2014;14:45780 .

10. Sammels E, Parys JB, Missiaen L, De Smedt H, Bultynck G. Intracellular Ca2+ storage in health and disease: a dynamic equilibrium. Cell Calcium 2010;47:297314.

11. Kiviluoto $\mathrm{S}$, Vervliet $\mathrm{T}$, Ivanova $\mathrm{H}$, Decuypere JP, De Smedt H, Missiaen L, et al. Regulation of inositol 1,4,5-trisphosphate receptors during endoplasmic reticulum stress. Biochim Biophys Acta 2013;1833:1612-24.

12. Cárdenas C, Foskett JK. Mitochondrial $\mathrm{Ca}(2+)$ signals in autophagy. Cell Calcium 2012;52:44-51.

13. Demaurex N, Frieden M. Measurements of the free luminal ER $\mathrm{Ca}(2+)$ concentration with targeted "cameleon" fluorescent proteins. Cell Calcium 2003;34:10919.

14. Ronco V, Potenza DM, Denti F, Vullo S, Gagliano G, Tognolina ML, et al. A novel Ca2+-mediated cross-talk between endoplasmic reticulum and acidic organelles: implications for NADP-dependent $\mathrm{Ca} 2+$ signalling. Cell Calcium 2015;57:89-100.

15. Maranto AR. Neuronal mapping: a photooxidation reaction makes Lucifer yellow useful for electron microscopy. Science 1982;217:953-5.

16. Sandell JH, Masland RH. Photoconversion of some fluorescent markers to a diaminobenzidine product. J Histochem Cytochem 1988;36:555-9.

17. Malatesta M, Giagnacovo M, Costanzo M, Conti B, Genta I, Dorati R, et al. Diaminobenzidine photoconversion is a suitable tool for tracking the intracellular location of fluorescently labelled nanoparticles at transmission electron microscopy. Eur J Histochem 2012; 56:e20.

18. Malatesta M, Pellicciari C, Cisterna B, Costanzo M, Galimberti V, Biggiogera M, et al. Tracing nanoparticles and photosensitizing molecules at transmission electron microscopy by diaminobenzidine photo-oxidation. Micron 2014;59:445.

19. Gerasimenko 0, Tepikin A. How to measure $\mathrm{Ca} 2+$ in cellular organelles? Cell Calcium 2005;38:201-11.

20. Solovyova N, Verkhratsky A. Measurement of free $\mathrm{Ca} 2+$ concentration in the lumen of neuronal endoplasmic reticulum. Cold Spring Harb Protoc 2010;2010:pdb. prot4783.

21. Moccia F, Dragoni S, Lodola F, Bonetti E, 
Bottino C, Guerra G, et al. Store-dependent $\mathrm{Ca}(2+)$ entry in endothelial progenitor cells as a perspective tool to enhance cell-based therapy and adverse tumour vascularization. Curr Med Chem 2012;19:5802-18.
22. Rizzuto R, Pozzan T. Microdomains of intracellular $\mathrm{Ca} 2+$ : molecular determinants and functional consequences. Physiol Rev 2006;86:369-408.

23. Guerrero-Hernandez A, Dagnino-Acosta A, Verkhratsky A. An intelligent sarco- endoplasmic reticulum $\mathrm{Ca} 2+$ store: release and leak channels have differential access to a concealed $\mathrm{Ca} 2+$ pool. Cell Calcium 2010;48:143-9. 